\title{
TANGENTIAL LIMITS OF FUNCTIONS ORTHOGONAL TO INVARIANT SUBSPACES
}

\author{
BY \\ DAVID PROTAS
}

\begin{abstract}
For any inner function $\varphi$, let $M^{1}$ be the orthogonal complement of $\varphi H^{2}$, in $H^{2}$, where $H^{2}$ is the usual Hardy space. The relationship between the tangential convergence of all functions in $M^{\perp}$ and the finiteness of certain sums and integrals involving $\varphi$ is studied. In particular, it is shown that the tangential convergence of all functions in $M^{\perp}$ is a stronger condition than the tangential convergence of $\varphi$, itself.
\end{abstract}

1. In this paper we study the boundary behavior of certain functions that are holomorphic in the unit disc $U$. For each $m>0$, each $\gamma \geqq 1$, and each point $\zeta$ on the boundary $\partial U$ of $U$, we let

$$
R(m, \zeta, \gamma)=\left\{z: 1-|z| \geqq m|\arg (\zeta z)|^{\gamma} ; 0<|z|<1\right\},
$$

where $\arg (\xi z)$ is always restricted to the interval $(-\pi, \pi]$. For the sake of convenience, set $R(m, \gamma)=R(m, 1, \gamma)$. Using Cargo's terminology [3], we say that a function $f$ defined on $U$ has a $T_{\gamma}$-limit at $\zeta$ if there exists a finite number $L$ such that, for each $m>0, f(z) \rightarrow L$ as $z \rightarrow \zeta$ through $R(m, \zeta, \gamma)$. Let us note that $f$ has a $T_{1^{-}}$ limit at $\zeta$ if and only if $f$ has a classical nontangential limit at $\zeta$.

Let

$$
B(z)=\prod_{n=1}^{\infty} \frac{\bar{a}_{n}}{\left|a_{n}\right|} \cdot \frac{a_{n}-z}{1-\bar{a}_{n} z},
$$

where $0<\left|a_{n}\right|<1$ and $\sum\left(1-\left|a_{n}\right|\right)<\infty$. B is holomorphic in $U$ and is called a Blaschke product. In [7], results of Cargo [3] and of Ahern and Clark [1] are used to obtain

THEOREM I. Let $B$ be a Blaschke product with zeros $\left\{a_{n}\right\}$ such that

$$
\sum_{n=1}^{\infty}\left(1-\left|a_{n}\right|^{2}\right) /\left|1-\xi a_{n}\right|^{\gamma}<\infty
$$

for some $\zeta \in \partial U$ and some $\gamma \geqq 1$. Then for any nonnegative integer $k$ with $k \leqq \gamma-1$, the $k$ th derivative of $B$, as well as the kth derivative of any subproduct of $B$, has a $T_{\gamma /(k+1)}$-limit at $\zeta$. In the case $k=0$, the limits are of modulus one. Moreover, for each

Received by the editors June 1, 1970 and, in revised form, March 1, 1971.

AMS 1969 subject classifications. Primary 3062; Secondary 3065, 3067.

Key words and phrases. Blaschke product, inner function, Hardy space, tangential limit, invariant subspace, boundary behavior. 
$m>0$, there exists a constant $C$ such that the kth derivative of $B$, as well as the kth derivative of any subproduct of $B$, is bounded in modulus by $C$ in the region $R(m, \zeta, \gamma /(k+1))$.

Linden and Somadasa show in [5] that the converse to Theorem I with $\gamma>1$ and $k=0$ does not hold. This has been expanded [7] to give

THEOREM II. Fix $\gamma>1$. For each $t>1$, there exists a Blaschke product $B$ with zeros $\left\{a_{n}\right\}$ such that $B$ and all its subproducts have $T_{\gamma}$-limits of modulus one at $\zeta=1$, but $\sum_{n=1}^{\infty}\left(1-\left|a_{n}\right|^{2}\right) /\left|1-a_{n}\right|^{t}=\infty$.

Let $H^{2}$ denote the usual Hardy class of functions holomorphic in $U$. A wellknown theorem of Beurling (see, for example, [8, p. 342]) states that any closed invariant subspace of $H^{2}$ is of the form $\varphi H^{2}$, where $\varphi$ is an inner function. That is, $\varphi$ is a bounded, holomorphic function in $U$ whose radial limits are of modulus one at almost all points of $\partial U$. In particular, any Blaschke product is inner. For any inner function $\varphi$, we let $\left(\varphi H^{2}\right)^{\perp}$ be the orthogonal complement of $\varphi H^{2}$ in $H^{2}$.

In $\S 2$, we give a result analogous to Theorem I relating $\left(B H^{2}\right)^{\perp}$ with the expression (1) for a Blaschke product $B$. In $\S 3$, a partial converse is obtained in contrast to Theorem II. Both of these results are shown to be best possible. $\$ 4$ is devoted to extending the work of the preceding sections to the case of a general closed invariant subspace of $H^{2}$.

This work represents part of the author's doctoral dissertation prepared at the University of Wisconsin under the direction of Professor P. R. Ahern, whose assistance the author gratefully acknowledges.

2. We start with

THEOREM 1. Let B be a Blaschke product with zeros $\left\{a_{n}\right\}$ such that

$$
\sum_{n}\left(1-\left|a_{n}\right|^{2}\right) /\left|1-\xi a_{n}\right|^{2 \gamma}<\infty
$$

for some $\zeta \in \partial U$ and $\gamma \geqq 1$. Then for each $f \in\left(B H^{2}\right)^{\perp}$ and each nonnegative integer $k$ with $k \leqq \gamma-1$, the $k$ th derivative of $f$ has a $T_{y /(k+1)}$-limit at $\zeta$.

Before beginning the proof, we note the result, which can be found in [4, Chapter VIII], that an inner function $\varphi$ is regular at a point of $\partial U$ if and only if each $f \in\left(\varphi H^{2}\right)^{\perp}$ is regular there. In particular, the conclusion of Theorem 1 holds for any finite Blaschke product. So, in our proof, we will assume that $B$ is an infinite Blaschke product.

Proof. Put $M=B H^{2}$. Let $B_{1}=1$, for $n=2,3, \ldots$, let $B_{n}$ be the subproduct of $B$ with zeros $a_{1}, \ldots, a_{n-1}$, and set

$$
h_{n}(z)=\left(1-\left|a_{n}\right|^{2}\right)^{1 / 2} B_{n}(z) /\left(1-\bar{a}_{n} z\right) .
$$

It is shown in [1] that $\left\{h_{n}\right\}$ is an orthonormal basis for $M^{\perp}$. Thus, replacing $B$ by the Blaschke product with zeros, $\left\{\xi a_{n}\right\}$ shows that there is no loss in generality in 
assuming that $\zeta=1$. Then, (2) implies that we can assume that $\arg a_{n}$ is never zero, also without loss of generality.

Let us pick any $f \in M^{\perp}$. Then for some $\left\{c_{n}\right\} \in l^{2}$,

$$
f(z)=\sum_{n=1}^{\infty} c_{n} h_{n}(z)
$$

for all $z \in U$. Fix $m>0$ and an integer $k$ with $0 \leqq k \leqq \gamma-1$. We wish to show first that the series $\sum\left|h_{n}^{(k)}(z)\right|^{2}$ converges uniformly in $R(m, \gamma /(k+1))$.

By Leibniz's rule,

$$
h_{n}^{(k)}(z)=\left(1-\left|a_{n}\right|^{2}\right)^{1 / 2} \sum_{j=0}^{k}\left(\begin{array}{c}
k \\
j
\end{array}\right) B_{n}^{(k-j)}(z) \frac{j ! \bar{a}_{n}^{j}}{\left(1-\bar{a}_{n} z\right)^{j+1}} .
$$

We know from Theorem I that there is a constant $C$ such that, for each $n$, $\left|B_{n}^{(k-j)}(z)\right| \leqq C$ for all $z$ in $R(m, 2 \gamma /(k+1)), j=0,1, \ldots, k$. So,

$$
\left|h_{n}^{(k)}(z)\right|^{2} \leqq C^{\prime} \sum_{j=0}^{2 k}\left(1-\left|a_{n}\right|\right) /\left|1-\bar{a}_{n} z\right|^{j+2}
$$

for all $z \in R(m, 2 \gamma /(k+1))$ and some constant $C^{\prime}$. On the other hand, it is shown in [3] that (2) implies

$$
\sum_{n=1}^{\infty} \frac{1-\left|a_{n}\right|}{w_{n}\left|\arg a_{n}\right|^{2 \gamma}}<\infty
$$

for some sequence $\left\{w_{n}\right\}$ such that $0<w_{n} \leqq 1$ and $w_{n} \rightarrow 0$ as $n \rightarrow \infty$. Fix $j=0,1, \ldots$, $2 k$, and put

$$
S_{j, n}=\left\{z:\left|z-a_{n}\right|<w_{n}^{1 /(j+2)}\left|\arg a_{n}\right|^{2 \gamma /(j+2)}\right\},
$$

$n=1,2, \ldots$ If $z \in U-S_{j, n}$, then

$$
\begin{aligned}
\left(1-\left|a_{n}\right|\right) /\left|1-\bar{a}_{n} z\right|^{j+2} & \leqq\left(1-\left|a_{n}\right|\right) /\left|z-a_{n}\right|^{j+2} \\
& \leqq\left(1-\left|a_{n}\right|\right) / w_{n}\left|\arg a_{n}\right|^{2 \gamma} .
\end{aligned}
$$

It is also shown in [3] that $R(m, \gamma /(k+1))$ intersects at most finitely many of the $S_{j, n}$. Thus,

$$
\left|h_{n}^{(k)}(z)\right|^{2} \leqq(2 k+1) C^{\prime}\left(1-\left|a_{n}\right|\right) / w_{n}\left|\arg a_{n}\right|^{2 \gamma}
$$

for all $z \in R(m, \gamma /(k+1))$ and all $n$ greater than some fixed integer. Therefore, by the Weierstrass $M$-test, $\sum\left|h_{n}^{(k)}(z)\right|^{2}$ converges uniformly in $R(m, \gamma /(k+1))$.

We can now conclude that $\left\{h_{n}^{(k)}(1)\right\}$ is square summable and that $\left\{h_{n}^{(k)}(z)\right\}$ approaches $\left\{h_{n}^{(k)}(1)\right\}$ in the $l^{2}$ norm as $z \rightarrow 1$ through $R(m, \gamma /(k+1))$. But,

$$
f^{(k)}(z)=\sum_{n=1}^{\infty} c_{n} h_{n}^{(k)}(z)
$$

So, as $z \rightarrow 1$ through $R(m, \gamma /(k+1)), f^{(k)}(z)$ has a finite limit since $\left\{h_{n}^{(k)}(z)\right\}$ approaches $\left\{h_{n}^{(k)}(1)\right\}$ weakly. This completes the proof. 
For any $z \in U$ and any nonnegative integer $k$, let $K_{z, k}$ be the function in $\left(B H^{2}\right)^{\perp}$ satisfying

$$
f^{(k)}(z)=\left(f, K_{z, k}\right)
$$

for all $f \in\left(B H^{2}\right)^{\perp}$. (For a general discussion of reproducing kernels, one can see [6].) Since $\left\{h_{n}\right\}$ is an orthonormal basis for $\left(B H^{2}\right)^{\perp}, K_{z, k}=\sum d_{n} h_{n}$ where $\left\|K_{z, k}\right\|^{2}$ $=\sum\left|d_{n}\right|^{2}$. As in [1], we see by setting $f=h_{n}$ in (4) that $\bar{d}_{n}=h_{n}^{(k)}(z)$. Thus, what we did in the proof above was to show that $\left\|K_{z, k}\right\|$ had a limit $\left\|K_{1, k}\right\|$ as $z \rightarrow 1$ through $R(m, \gamma /(k+1)), m>0$.

The special case of Theorem 1 corresponding to $\gamma=1$ can be found, along with its converse, in [1]. Let us now prove that Theorem 1 for general $\gamma \geqq 1$ is best possible in a natural sense.

EXAMPLE 1. Choose a number $\gamma \geqq 1$, and let $k$ be any nonnegative integer with $k \leqq \gamma-1$. For each $t>\gamma /(k+1)$, there exists a Blaschke product $B$ with zeros $\left\{a_{n}\right\}$ such that (2) holds with $\zeta=1$, but $f^{(k)}$ does not have a $T_{t}$-limit at $\zeta=1$ for each $f$ in some dense $G_{\delta}$ subset of $\left(B H^{2}\right)^{\perp}$.

Proof. We can assume without loss of generality that $t \leqq 2 \gamma /(k+1)$. Let us choose a positive number $\alpha$ such that $1 /(2 k+2) \alpha<t-\gamma /(k+1)$. Then, $1+2 \gamma \alpha<(2 k+2) t \alpha$, and we can choose a number $\beta$ so that $1+2 \gamma \alpha<\beta<(2 k+2) t \alpha$. Also, we require that $\beta$ be chosen so that $\beta>2 k t \alpha$. Now put $\arg a_{n}=n^{-\alpha}$ and $\left|a_{n}\right|=1-n^{-\beta}$ for $n=2,3, \ldots$

We have

$$
\sum_{n=2}^{\infty}\left(1-\left|a_{n}\right|\right) /\left|\arg a_{n}\right|^{2 \gamma}=\sum_{n=2}^{\infty} 1 / n^{\beta-2 \gamma \alpha}<\infty
$$

since $\beta>1+2 \gamma \alpha$. Now (2) with $\zeta=1$ follows since $\sin \left|\arg a_{n}\right| \leqq\left|1-a_{n}\right|$.

Let $K_{z, k}$ be the function given by (4), and fix $m>0$. Because of the uniform boundedness theorem, we shall be done if we can show that $\left\|K_{z, k}\right\|$ is unbounded in $R(m, t)$.

Define $z_{n} \in R(m, t)$ by $\arg z_{n}=\arg a_{n},\left|z_{n}\right|=1-m\left|\arg a_{n}\right|^{t}$, for $n=2,3, \ldots$ We shall show that $\left|h_{n}^{(k)}\left(z_{n}\right)\right| \rightarrow \infty$ as $n \rightarrow \infty$, using formula (3). A simple calculation gives

$$
\frac{1-\bar{a}_{n} z_{n}}{\left(1-\left|a_{n}\right|\right)^{1 /(2 j+2)}}=\frac{n^{-\beta}+m n^{-t \alpha}-m n^{-(\beta+t \alpha)}}{n^{-\beta /(2 j+2)}}
$$

and so as $n \rightarrow \infty,\left(1-\left|a_{n}\right|^{2}\right)^{1 / 2} /\left(1-\bar{a}_{n} z_{n}\right)^{j+1}$ approaches 0 for $j=0,1, \ldots, k-1$ (if $k>0)$ and approaches $\infty$ for $j=k$ since $2 k t \alpha<\beta<(2 k+2) t \alpha$. Furthermore, (2) holds and $t \leqq 2 \gamma /(k+1)$. So Theorem I implies that $\left|B_{n}\left(z_{n}\right)\right| \rightarrow 1$ since $\left|B_{n}\left(z_{n}\right)\right|$ $\geqq\left|B\left(z_{n}\right)\right|$, and that $\left|B_{n}^{(k-j)}\left(z_{n}\right)\right|$ remains bounded as $n \rightarrow \infty, j=0,1, \ldots, k-1$ (if $k>0)$. Thus, $B_{n}^{(k-j)}\left(z_{n}\right)\left(1-\left|a_{n}\right|^{2}\right)^{1 / 2} /\left(1-\bar{a}_{n} z_{n}\right)^{j+1}$ approaches 0 for $j=0,1, \ldots, k-1$ (if $k>0$ ) and approaches $\infty$ for $j=k$. So, $\left|h_{n}^{(k)}\left(z_{n}\right)\right| \rightarrow \infty$ as $n \rightarrow \infty$. Therefore, $\left\|K_{z_{n}, k}\right\| \rightarrow \infty$ as $n \rightarrow \infty$. 
3. We now prove a partial converse to Theorem 1 with $k=0$. Actually, we prove a little more.

THEOREM 2. Let $B$ be a Blaschke product with zeros $\left\{a_{n}\right\}$ and choose any $\zeta \in \partial U$, $\gamma \geqq 1$. Then, if for some $m>0$, each function in $\left(B H^{2}\right)^{\perp}$ is bounded in $R(m, \zeta, \gamma)$, it follows that

$$
\sum_{n=1}^{\infty}\left(1-\left|a_{n}\right|^{2}\right) /\left|1-\xi a_{n}\right|^{t}<\infty
$$

for all $t<\gamma+1$.

Proof. It is clear that there is no loss of generality in assuming that $a_{n} \rightarrow \zeta$ as $n \rightarrow \infty$ and that $\zeta=1$. Fix $m>0$ such that each function in $\left(B H^{2}\right)^{\perp}$ is bounded in $R(m, \gamma)$. The uniform boundedness theorem implies that $\left\|K_{z}\right\|$ is bounded as $z$ ranges over $R(m, \gamma)$, where $K_{z}=K_{z, 0}$. We have

$$
\left\|K_{z}\right\|^{2}=\sum_{n=1}^{\infty}\left|B_{n}(z)\right|^{2}\left(1-\left|a_{n}\right|^{2}\right) /\left|1-\bar{a}_{n} z\right|^{2} .
$$

On the other hand, $K_{z}(\lambda)=(1-\bar{B}(z) B(\lambda)) /(1-\bar{z} \lambda)$ and so

$$
\left\|K_{z}\right\|^{2}=\left(1-|B(z)|^{2}\right) /\left(1-|z|^{2}\right)
$$

since $\left\|K_{z}\right\|^{2}=K_{z}(z)$. So, $\left\|K_{z}\right\|$ being bounded in $R(m, \gamma)$ implies that $|B(z)| \rightarrow 1$ as $z \rightarrow 1$ through $R(m, \gamma)$. Thus, there exists a constant $C$ such that

$$
\sum_{n=1}^{\infty}\left(1-\left|a_{n}\right|^{2}\right) /\left|1-\bar{a}_{n} z\right|^{2} \leqq C
$$

for all $z \in R(m, \gamma)$. In particular, $\left(1-\left|a_{n}\right|^{2}\right)^{-1} \leqq C$ for each $n$ with $a_{n} \in R(m, \gamma)$ and so, there is a number $n_{0}$ such that $1-\left|a_{n}\right|<m\left|\arg a_{n}\right|^{\gamma}$ (and $\left|\arg a_{n}\right|^{\gamma-1}<1 / m$ if $\gamma>1$ ) for all $n \geqq n_{0}$.

Next, let us put $A=\left\{n: \arg a_{n}>0\right.$ and $\left.n \geqq n_{0}\right\}$. Denote by $\partial R^{+}$that part of $\partial R(m, \gamma)$ which lies in the upper half-plane. For any $n \in A$ and $z \in \partial R^{+}$we have, by the law of cosines,

$$
\begin{aligned}
\left|1-\bar{a}_{n} z\right|^{2} & =\left(1-\left|a_{n}\right||z|\right)^{2}+4\left|a_{n}\right||z| \sin ^{2} \frac{1}{2}\left(\arg a_{n}-\arg z\right) \\
& \leqq\left(1-\left|a_{n}\right|+m|\arg z|^{\gamma}\right)^{2}+\left(\arg a_{n}-\arg z\right)^{2} \\
& \leqq\left(m\left|\arg a_{n}\right|^{\gamma}+m|\arg z|^{\gamma}\right)^{2}+\left(\arg a_{n}-\arg z\right)^{2}
\end{aligned}
$$

For any $n \in A$, set

$$
E_{n}=\left\{u>0: \arg a_{n}-m\left|\arg a_{n}\right|^{\gamma} \leqq u \leqq \arg a_{n}\right\} .
$$

Then whenever $z \in \partial R^{+}$has $\arg z \in E_{n}$, we get

$$
\left|1-\bar{a}_{n} z\right|^{2} \leqq 5 m^{2}\left|\arg a_{n}\right|^{2 \gamma} \quad(n \in A) .
$$


Setting $u=\arg z$, we can think of $z \in \partial R^{+}$as a function of $u$. Choose any $p$ with $0<p<1$ and assume that $m \leqq \pi^{-\gamma}$. Then (5) implies

$$
\int_{0}^{\pi} \sum_{n \in A} \frac{1-\left|a_{n}\right|^{2}}{\left|1-\bar{a}_{n} z\right|^{2}} u^{-p} d u \leqq C \pi^{1-p} /(1-p)
$$

where $z$ is considered to be a function of $u$. So,

$$
\sum_{n \in A} \int_{E_{n}} \frac{1-\left|a_{n}\right|^{2}}{\left|1-\bar{a}_{n} z\right|^{2}} u^{-p} d u \leqq C \pi^{1-p} /(1-p)
$$

and then

$$
\sum_{n \in A} \int_{E_{n}} \frac{1-\left|a_{n}\right|^{2}}{5 m^{2}\left|\arg a_{n}\right|^{2 \gamma}} u^{-p} d u \leqq C \pi^{1-p} /(1-p)
$$

An elementary computation shows that there is a constant $C_{0}>0$ such that $\int_{E_{n}} u^{-p} d u \geqq C_{0}\left|\arg a_{n}\right|^{\gamma-p}$ for large enough $n$. So we conclude that

$$
\sum_{n \in A}\left(1-\left|a_{n}\right|^{2}\right) /\left|\arg a_{n}\right|^{\gamma+p}<\infty \text {. }
$$

Applying the preceding work to the Blaschke product with zeros $\left\{\bar{a}_{n}\right\}$ and noting that $\arg a_{n} \neq 0$ whenever $n \geqq n_{0}$, we get

$$
\sum_{n=n_{0}}^{\infty}\left(1-\left|a_{n}\right|^{2}\right) /\left|\arg a_{n}\right|^{\gamma+p}<\infty .
$$

The desired result follows since $\sin \left|\arg a_{n}\right| \leqq\left|1-a_{n}\right|$. If $m>\pi^{-\gamma}$, integrate (5) over $\left(0, m^{-1 / \gamma}\right)$ instead of $(0, \pi)$. Then proceed as above.

Comparing Theorem 2 in the case $\gamma>1$ with Theorem II, we see that the tangential limit behavior of the functions in $\left(B H^{2}\right)^{\perp}$ tells us more about the zeros of $B$ than does the tangential limit behavior of $B$, itself, along with all its subproducts. We shall now prove that Theorem 2 with $\gamma>1$ is best possible. The theorem of Ahern and Clark [1] referred to just before the last example shows that Theorem 2 can be slightly improved in the case $\gamma=1$. This improvement also can be gotten from formula (5).

EXAMPLE 2. For any $\gamma>1$, there exists a Blaschke product $B$ with zeros $\left\{a_{n}\right\}$ such that each function in $\left(B H^{2}\right)^{\perp}$ has a $T_{\gamma}$-limit at $\zeta=1$, but

$$
\sum_{n=1}^{\infty}\left(1-\left|a_{n}\right|^{2}\right) /\left|1-a_{n}\right|^{\gamma+1}=\infty
$$

Proof. For $n=2,3, \ldots$, let $\arg a_{n}=n^{-\alpha}$ and $\left|a_{n}\right|=1-n^{-\beta}$, where $\alpha=1 /(\gamma-1)$ and $\beta=2 \gamma /(\gamma-1)$. Then,

$$
\sum_{n=2}^{\infty}\left(1-\left|a_{n}\right|\right) /\left|\arg a_{n}\right|^{\gamma+1}=\sum_{n=2}^{\infty} 1 / n^{\beta-(\gamma+1) \alpha}=\infty
$$

since $\beta-(\gamma+1) \alpha=1$. So, (6) follows from an argument given in [3]. 
Fix any $m>0$ small enough that $1-m 2^{-\alpha} \geqq \frac{3}{4}$ and $m<\alpha / 4$. Then for each $j=2,3$, $\ldots$, define $z_{j} \in R(m, \gamma)$ by $\arg z_{j}=\arg a_{j}$ and $\left|z_{j}\right|=1-m\left|\arg a_{j}\right|^{\nu}$. We are going to show that the $H^{2}$ norm of $K_{z}$ is bounded in $R(m, \gamma)$ by first considering $z=z_{j}$.

By the law of cosines,

$$
\begin{aligned}
\left|1-\bar{a}_{n} z_{j}\right|^{2} & =\left(1-\left|a_{n}\right|\left|z_{j}\right|\right)^{2}+4\left|a_{n}\right|\left|z_{j}\right| \sin ^{2} \frac{1}{2}\left(\arg a_{n}-\arg z_{j}\right) \\
& \geqq\left(n^{-\beta}+m j^{-\gamma \alpha}-m j^{-\gamma \alpha} n^{-\beta}\right)^{2}+\frac{1}{2}\left(n^{-\alpha}-j^{-\alpha}\right)^{2} .
\end{aligned}
$$

In particular, $\left(1-\left|a_{j}\right|\right) /\left|1-\bar{a}_{j} z_{j}\right|^{2} \leqq 1 / m^{2}$ since $\beta=2 \gamma \alpha$. If $j<n \leqq 2 j$, an elementary calculation, using the factorization of $x^{k}-y^{k}$ ( $k$ a positive integer), shows that $2^{\alpha} \alpha^{-1} n^{1-\alpha}\left(n^{\alpha}-j^{\alpha}\right) \geqq n-j$ for all rational $\alpha>0$ and so for all $\alpha>0$. Also, note that $\beta-2 \alpha=2$. So,

$$
\begin{aligned}
\sum_{n=j+1}^{2 j} \frac{1-\left|a_{n}\right|}{\left|1-\bar{a}_{n} z_{j}\right|^{2}} & \leqq 2 \sum_{n=j+1}^{2 j} \frac{j^{2 \alpha} n^{-2}}{\left(n^{\alpha}-j^{\alpha}\right)^{2}} \leqq \frac{2^{2 \alpha+1}}{\alpha^{2}} \sum_{n=j+1}^{2 j} \frac{j^{2 \alpha} n^{-2 \alpha}}{(n-j)^{2}} \\
& \leqq \frac{2^{2 \alpha+1}}{\alpha^{2}} \sum_{n=j+1}^{2 j} 1 /(n-j)^{2} \leqq \frac{2^{2 \alpha+1}}{\alpha^{2}} \sum_{n=1}^{\infty} 1 / n^{2} .
\end{aligned}
$$

Likewise, letting $p$ be the greatest integer in $j / 2$, we get

$$
\sum_{n=p+1}^{j-1}\left(1-\left|a_{n}\right|\right) /\left|1-\bar{a}_{n} z_{j}\right|^{2} \leqq\left(2^{\beta+1} / \alpha^{2}\right) \sum_{n=1}^{\infty} 1 / n^{2} .
$$

Also,

$$
\sum_{n=2 j+1}^{\infty} \frac{1-\left|a_{n}\right|}{\left|1-\bar{a}_{n} z_{j}\right|^{2}} \leqq 2 \sum_{n=2 j+1}^{\infty} \frac{j^{2 \alpha} n^{-2}}{\left((2 j)^{\alpha}-j^{\alpha}\right)^{2}} \leqq \frac{2}{\left(2^{\alpha}-1\right)^{2}} \sum_{n=1}^{\infty} 1 / n^{2} ;
$$

and similarly,

$$
\sum_{n=2}^{p}\left(1-\left|a_{n}\right|\right) /\left|1-\bar{a}_{n} z_{j}\right|^{2} \leqq 2\left(1-2^{-\alpha}\right)^{-2} \sum_{n=1}^{\infty} 1 / n^{2} .
$$

Thus, we conclude that

$$
\sum_{n=2}^{\infty}\left(1-\left|a_{n}\right|\right) /\left|1-\bar{a}_{n} z\right|^{2}
$$

is bounded for $z=z_{j}$, where $2 \leqq j<\infty$.

For $j=2,3, \ldots$, let $w_{j}$ be the value of $z \in R(m, \gamma)$ which minimizes $\left|1-\bar{a}_{j} z\right|$ $=\left|a_{j}\right| \cdot\left|\bar{a}_{j}^{-1}-z\right|$. The existence and uniqueness of each $w_{j}$ is ensured by the convexity of $R(m, \gamma) \cap\{z: \operatorname{Im} z>0\}$. Since $\partial R(m, \gamma)$ is tangent to $\partial U$, the law of sines implies that there is an integer $n_{0}$ such that $\left|1-\bar{a}_{j} z_{j}\right| \leqq 2\left|1-\bar{a}_{j} w_{j}\right|$ for all $j \geqq n_{0}$. Next we observe that $w_{j}$ lies on $\partial R^{+}$between $z_{j}$ and 1 . By l'Hospital's rule, when $j$ is sufficiently large,

$$
\frac{\left|1-\bar{a}_{j} z_{j}\right|}{\left|1-\bar{a}_{j} z_{j+1}\right|} \leqq \frac{4 m j^{-\gamma \alpha}}{j^{-\alpha}-(j+1)^{-\alpha}}=\frac{4 m j^{-1}}{1-(1+1 / j)^{-\alpha}} \sim \frac{4 m}{\alpha}<1
$$

since $\alpha(\gamma-1)=1$ and $m<\alpha / 4$. So, there is an integer $n_{1} \geqq n_{0}$ such that $w_{j}$ lies between 
$z_{j}$ and $z_{j+1}$ for all $j \geqq n_{1}$. Pick $z \in \partial R^{+}$and suppose $z$ lies between $z_{j}$ and $z_{j+1}$, $j \geqq n_{1}$. Then the preceding work gives

$$
\sum_{n=2}^{\infty} \frac{1-\left|a_{n}\right|}{\left|1-\bar{a}_{n} z\right|^{2}} \leqq \sum_{n=2}^{j-1} \frac{1-\left|a_{n}\right|}{\left|1-\bar{a}_{n} z_{j}\right|^{2}}+\frac{4\left(1-\left|a_{j}\right|\right)}{\left|1-\bar{a}_{j} z_{j}\right|^{2}}+\sum_{n=j+1}^{\infty} \frac{1-\left|a_{n}\right|}{\left|1-\bar{a}_{n} z_{j+1}\right|^{2}} .
$$

So, (7) is bounded on $\partial R^{+}$.

Let us choose any $z \in R(m, \gamma)$ with $z=x+i y, x>0, y>0$. Then, let $z^{\prime}=x+i y^{\prime}$ and $z^{\prime \prime}=x^{\prime \prime}+i y$ be on $\partial R(m, \gamma)$ with $y^{\prime} \geqq y$ and $x^{\prime \prime} \geqq x$. For any $a_{n}$, put $\bar{a}_{n}^{-1}=b_{n}+i c_{n}$. Either $b_{n} \geqq\left(x+x^{\prime \prime}\right) / 2$ or $c_{n} \geqq\left(y+y^{\prime}\right) / 2$. If $b_{n} \geqq\left(x+x^{\prime \prime}\right) / 2$, then $\left|\bar{a}_{n}^{-1}-z\right| \geqq\left|\bar{a}_{n}^{-1}-z^{\prime \prime}\right|$ and so $\left|1-\bar{a}_{n} z\right| \geqq\left|1-\bar{a}_{n} z^{\prime \prime}\right|$. Likewise, $\left|1-\bar{a}_{n} z\right| \geqq\left|1-\bar{a}_{n} z^{\prime}\right|$ if $c_{n} \geqq\left(y+y^{\prime}\right) / 2$. Finally, choose any $z \in R(m, \gamma)$ with $z=x+i y, x>0, y<0$. Then, $\bar{z}=x+i(-y) \in R(m, \gamma)$ and $\left|1-\bar{a}_{n} z\right| \geqq\left|1-\bar{a}_{n} \bar{z}\right|$. We conclude that (7) is bounded in $R(m, \gamma)$, and so, $\left\|K_{z}\right\|$ is bounded in $R(m, \gamma)$. (That $\left\|K_{z}\right\|$ is bounded for $z \in R(m, \gamma)$ with $y=0$ can be seen from the continuity of $\left\|K_{z}\right\|^{2}$.)

Let $z$ approach 1 through $R(m, \gamma)$. Since the closed unit ball in a Hilbert space is weakly compact, there is a weak limit point given by, say, $K_{1} \in\left(B H^{2}\right)^{\perp}$. We note that $K_{1}$ is unique since it is determined by $\left(h_{n}, K_{1}\right), n=2,3, \ldots$, and each $h_{n}$ is continuous on the closure of $U$. Therefore, $K_{z} \rightarrow K_{1}$ weakly as $z \rightarrow 1$ through $R(m, \gamma)$ and we are done.

Let us note that the Blaschke product in the last example has the property that $\left\|K_{z}\right\|$ does not approach $\left\|K_{1}\right\|$ as $z \rightarrow 1$ through $R(m, \gamma)$ for some $m>0$ even though each $f \in(B H)^{\perp}$ has a $T_{\gamma}$-limit. This is seen by checking that $\left(1-\left|a_{j}\right|\right) /\left|1-\bar{a}_{j} z_{j}\right|^{2}$ $\rightarrow 1 / m^{2}$ as $j \rightarrow \infty$, for then $\left\|K_{z,}\right\|$ cannot approach $\left\|K_{1}\right\|$ if $m$ is chosen small enough.

So it is natural to ask whether we can get something more closely resembling a converse to Theorem 1 with $k=0$ by assuming that $\left\|K_{z}\right\|$ has a $T_{\gamma}$-limit. A slight alteration of the last example shows that nothing significant can be attained by this approach. Suppose $\gamma>1$ and $t>\gamma+1$. Then by choosing $(\gamma-1)^{-1}<\alpha$ $<(2 \gamma-t)^{-1}$ and $2 \gamma \alpha<\beta \leqq 1+t \alpha$, we get a Blaschke product $B$ such that $\left\|K_{z}\right\|$ has a $T_{\gamma}$-limit at $\zeta=1$ but $\sum\left(1-\left|a_{n}\right|^{2}\right) /\left|1-a_{n}\right|^{t}=\infty$. The proof is very similar to that of Example 2.

4. In this section we extend our results to arbitrary closed invariant subspaces of $H^{2}$. Since the proofs are quite similar to those given in the last two sections, we will only give brief sketches.

If $\varphi$ is an inner function, then $\varphi=M B s$ where $M$ is a monomial, $B$ is a Blaschke product with zeros $\left\{a_{n}\right\}\left(a_{n} \neq 0\right)$, and $s$ is a singular inner function. That is,

$$
s(z)=\exp \left\{-\int_{0}^{2 \pi}\left(e^{i \theta}+z\right) /\left(e^{i \theta}-z\right) d \sigma(\theta)\right\},
$$

where $\sigma$ is a finite positive singular measure. The function $s$, in turn, can be factored into the product of two singular inner functions, $s_{c}$ and $s_{a}$, corresponding to, respectively, a continuous measure $\sigma_{c}$ and a purely atomic measure $\sigma_{a}$ with $\sigma=\sigma_{c}+\sigma_{a}$. 
THEOREM 3. Let $\varphi=M B$ s be an inner function. Suppose that

$$
\sum_{n}\left(1-\left|a_{n}\right|^{2}\right) /\left|1-\zeta a_{n}\right|^{2 \gamma}<\infty
$$

and

$$
\int_{0}^{2 \pi}\left|1-\zeta e^{i \theta}\right|^{-2 \gamma} d \sigma(\theta)<\infty
$$

for some fixed $\zeta \in \partial U, \gamma \geqq 1$. Then for each $f \in\left(\varphi H^{2}\right)^{\perp}$ and each nonnegative integer $k$ with $k \leqq \gamma-1$, the $k$ th derivative of $f$ has a $T_{\gamma /(k+1)}$-limit at $\zeta$.

Proof. It is clear that (9) holds with $\sigma$ replaced by either $\sigma_{a}$ or $\sigma_{c}$. So, a theorem in [7] analogous to Theorem I states that $s_{c}^{(j)}$ and $s_{a}^{(j)}$ have $T_{2 y /(j+1)}$-limits, $j=0,1$, $\ldots, k$. It is easy to check with the aid of the Cauchy formula that $\left(M H^{2}\right)^{\perp}$ is just the space of all polynomials of degree less than the degree of $M$. Also, a lemma in [2] states that if $\varphi_{1}$ and $\dot{\varphi}_{2}$ are inner functions, then

$$
\left(\varphi_{1} \varphi_{2} H^{2}\right)^{\perp}=\left(\varphi_{1} H^{2}\right)^{\perp} \oplus \varphi_{1}\left(\varphi_{2} H^{2}\right)^{\perp} .
$$

So this, along with Theorem I and Theorem 1, implies that we need only show that the conclusion holds for functions in $\left(s_{c} H^{2}\right)^{\perp}$ and $\left(s_{a} H^{2}\right)^{\perp}$.

It is proven in [2] that if $f \in\left(s_{c} H^{2}\right)^{\perp}$, then

$$
f(z)=\int_{0}^{2 \pi} c(t) 2^{1 / 2} s_{c, t}(z)\left(1-e^{-i t} z\right)^{-1} d \sigma_{c}(t)
$$

where $c \in L^{2}(d \sigma)$ and $s_{c, t}$ is the inner function given by

$$
s_{c, t}(z)=\exp \left\{-\int_{0}^{t}\left(e^{i \theta}+z\right) /\left(e^{i \theta}-z\right) d \sigma_{c}(\theta)\right\} .
$$

Then, the conclusion for $\left(s_{c} H^{2}\right)^{\perp}$ follows from (9) with $\sigma$ replaced by $\sigma_{c}$ in the same way that the conclusion of Theorem 1 followed from (2) using the basis $\left\{h_{n}\right\}$. The proof is so similar that it will be omitted. Likewise, it is shown in [2] that if $f \in\left(s_{a} H^{2}\right)^{\perp}$ and $\sigma_{a}$ consists of masses $r_{n}$ at points $\theta_{n}$, then

$$
f(z)=\int_{0}^{\infty} c(t) 2^{1 / 2} s_{a, t}(z)\left(1-\exp \left(-i \theta_{N+1}\right) z\right)^{-1} d \tau(t)
$$

where $N$ is the greatest integer in $t, \tau$ is the measure on $(0, \infty)$ given by $d \tau(t)$ $=r_{N+1} d t$ on $(N, N+1], N=0,1, \ldots$, and $s_{a, t}$ is the inner function given by

$$
s_{a, t}(z)=\exp \left\{-\sum_{j=1}^{N} r_{j} \frac{\exp \left(i \theta_{j}\right)+z}{\exp \left(i \theta_{j}\right)-z}-(t-N) r_{N+1} \frac{\exp \left(i \theta_{N+1}\right)+z}{\exp \left(i \theta_{N+1}\right)-z}\right\} .
$$

The proof for $\left(s_{a} H^{2}\right)^{\perp}$ follows as in the previous cases. 
THEOREM 4. Let $\varphi=M B s$ be an inner function and choose any $\zeta \in \partial U, \gamma \geqq 1$. Then, if for some $m>0$, each function in $\left(\varphi H^{2}\right)^{\perp}$ is bounded in $R(m, \zeta, \gamma)$, it follows that

$$
\sum_{n}\left(1-\left|a_{n}\right|^{2}\right) /\left|1-\zeta a_{n}\right|^{t}<\infty
$$

and

$$
\int_{0}^{2 \pi}\left|1-\zeta e^{i \theta}\right|^{-t} d \sigma(\theta)<\infty
$$

for all $t<\gamma+1$.

Proof. Each function in $\left(B H^{2}\right)^{\perp},\left(s_{c} H^{2}\right)^{\perp}$, and $\left(s_{a} H^{2}\right)^{\perp}$ is bounded in $R(m, \zeta, \gamma)$ since all of these spaces are contained in $\left(\varphi H^{2}\right)^{\perp}$. So (10) holds by Theorem 2 . In the case of $\left(s_{c} H^{2}\right)^{\perp}$, we have for the function $K_{z}$ given by $f(z)=\left(f, K_{z}\right)$ that

$$
\left\|K_{z}\right\|^{2}=2 \int_{0}^{2 \pi}\left|s_{c, t}(z)\right|^{2} /\left|1-e^{-i t} z\right|^{2} d \sigma_{c}(t) .
$$

Then, (11) with $\sigma$ replaced by $\sigma_{c}$ follows. The proof is very similar to the proof of Theorem 2 and will be omitted. Likewise, in the case of $\left(s_{a} H^{2}\right)^{\perp}$, we have

$$
\left\|K_{z}\right\|^{2}=2 \int_{0}^{\infty}\left|s_{a, t}(z)\right|^{2} /\left|1-\exp \left(-i \theta_{N+1}\right) z\right|^{2} d \tau(t)
$$

and (11) with $\sigma$ replaced by $\sigma_{a}$ follows.

\section{REFERENCES}

1. P. R. Ahern and D. N. Clark, Radial limits and invariant subspaces, Amer. J. Math. 92 (1970), 332-342. MR 41 \#7117.

2. - On functions orthogonal to invariant subspaces, Acta Math. 124 (1970), 191-204. MR 41 \#8981a.

3. G. T. Cargo, Angular and tangential limits of Blaschke products and their successive derivatives, Canad. J. Math. 14 (1962), 334-348. MR 25 \#204.

4. H. Helson, Lectures on invariant subspaces, Academic Press, New York, 1964. MR 30 \#1409.

5. C. N. Linden and H. Somadasa, On tangential limits of Blaschke products, Arch. Math. (Basel) 18 (1967), 416-424. MR 38 \#2306.

6. H. Meschkowski, Hilbertsche Räume mit Kernfunktion, Die Grundlehren der math. Wissenschaften, Band 113, Springer-Verlag, Berlin, 1962. MR 25 \#4326.

7. D. Protas, Tangential limits of Blaschke products and functions of bounded characteristic, Arch. Math. (Basel) (to appear).

8. W. Rudin, Real and complex analysis, McGraw-Hill, New York, 1966. MR 35 \#1420.

Department of Mathematics, San Fernando Valley State College, Northridge, California 91324 